\title{
Switching Strategy for Sensor Fault Tolerant Vector Control of Doubly Fed Induction Machines
}

\author{
Mónica E. Romero
}

\author{
María M. Seron
}

\begin{abstract}
In this paper we propose a multisensor switching strategy for fault tolerant control of rotor wound induction motors operating as stand alone generators. The proposed strategy combines three current sensors and associated observers that estimate the stator flux. The estimates provided by the observers are compared at each sampling time by a switching mechanism, which selects the sensors-observer pair with the smallest error between the estimated flux magnitude and a desired flux reference. The estimates provided by the selected pair are used to implement a vector control law. The switching selection is such that measurements from faulty current sensors are automatically avoided by the switching mechanism, thus maintaining good performance levels even in the presence of a faulty sensor. Simulation results under realistic conditions illustrate the effectiveness of the scheme.
\end{abstract}

\section{INTRODUCTION}

In this paper we investigate the use of multisensor switching strategies [1], [2] in an adjustable speed generator (ASG) control scheme of a wound rotor induction motor (WRIM) operating as a stand alone generation system. The ASG control scheme consists of the WRIM employed as a doubly fed induction generator (DFIG) with a four-quadrant ac-to-ac converter connected to the rotor windings [3].

DFIMs have become a widely used generator type in wind energy conversion. DFIGs are also used in pump storage plants, in flywheel energy storage, as well as in marine and aviation systems [4]. In all such applications, faults in any of the components of the feedback loop (the generator itself, the driving circuits, sensors, actuators, etc.) can seriously deteriorate the overall system performance. It is thus crucial, in order to avoid failure of the whole system, to design control strategies that are "fault tolerant", that is, strategies which are capable to maintain stability and good performance levels even under the occurrence of severe component faults.

In this paper, we propose the use of a sensor fault tolerant control strategy that combines rotor current sensors, a bank of three stator flux observers, an estimate switching mechanism, and vector control of the induction machine, as illustrated in Figure 1. Each observer provides estimates of the stator flux based on noisy measurements of two phase currents. At each sampling time, the switching mechanism

M. Romero is with the Laboratorio de Sistemas Dinámicos y Procesamiento de la Información, Universidad Nacional de Rosario, Riobamba 245 bis, 2000 Rosario, Argentina (mromero@fceia.unr.edu.ar)

M. Seron is with the Centre for Complex Dynamic Systems and Control, The University of Newcastle, Callaghan NSW 2308, Australia (maria.seron@newcastle.edu.au)

This work has been partially supported by the Agencia Nacional de Promoción Científica y Tecnológica, Argentina, under project PICT 20080650 (FONCyT). selects the sensors-observer pair with the smallest error between the estimated stator flux magnitude and a desired flux reference, and passes the selected state estimates to the vector controller. We consider the vector control technique presented in [5]. The implementation of this technique requires measurements or estimates of the rotor speed, rotor currents and stator flux. We will assume here that the rotor speed, stator voltages and rotor currents are measured. In contrast, the stator flux is estimated from the available measurements by means of flux observers of the form proposed by [6].

We can find in the literature different approaches for fault detection in DFIG systems. A fault diagnosis system based on the generalised observer scheme and a statistical decision system was proposed in [7] for monitoring the stator current and voltage sensors of a DFIG under linear quadratic Gaussian control. In [8], a field-programmable-gate-array-based grid-side-converter current sensor fault tolerant control for WECS with DFIG is proposed. Although the detection mechanism is initially simple, there are several disadvantages that must be corrected by adding new blocks and, in consequence, simplicity is lost. The work [9] presents a performance study of diagnosis methods for IGBT open circuit faults in three phase voltage source inverters for AC variable speed drives. In [4] a bilinear observer is presented to detect current sensor faults in a DFIG. Four sensors, two measuring stator currents and two measuring rotor currents, are necessary to implement the control strategy. In [10] and [11] a bank of three different observers based on different measurements (stator currents, rotor currents and stator voltages) is proposed, which become active depending on the presented fault. In those works it is not clear which pair of measurements-observer are used in healthy operation conditions and (similarly to [4]) the motivation for using four currents sensors to implement the control strategy.

Departing from the aforementioned approaches, our proposed scheme achieves faulty sensor detection and isolation and automatic controller reconfiguration "implicitly" by guaranteeing that the switching cost avoids selecting faulty sensors. Indeed, our proposed strategy is such that, when a current sensor fails, the observers that use measurements from the faulty sensor are automatically avoided by the switching mechanism, hence maintaining good performance levels even under severe sensor faults. Applications of a similar switching methodology to vector control of induction motors was presented in [12] and [13] using, respectively, field oriented control and direct flux and torque control techniques. A sensorless version was proposed in [14].

The remainder of the paper is organised as follows. 


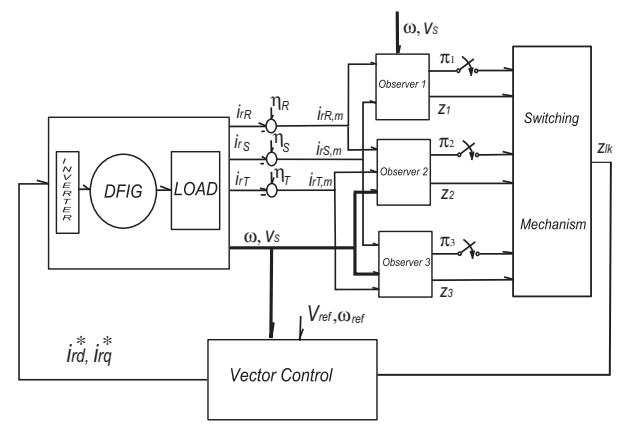

Fig. 1. Fault tolerant control of a DFIG. Note that the rotor speed, $\omega$ and the stator voltage, $V_{s}=\left[v_{s q} v_{s d}\right]^{T}$, are measured for their use in the observers and the vector control strategy.

Section II presents the model of the wound rotor induction motor. Section III briefly explains the vector control technique for DFIG employed in this paper. Section IV describes the sensing scheme and flux observers. In Section $\mathrm{V}$ we present the proposed estimate switching mechanism and controller implementation. Section VI analyses the closedloop system performance under healthy operation of all sensors. Section VII analyses the system performance under an abrupt fault of a current sensor and explains the fault tolerance principle. Section VIII illustrates the proposed approach by means of a simulation example. Finally, the paper is concluded in Section IX.

\section{MODEL OF THE WRIM}

The wound rotor induction motor (WRIM) can be described by the following equations using a $d q$ reference frame rotating at an arbitrary speed $\omega_{d q}[15]:^{1}$

$$
\begin{aligned}
\frac{d x}{d t} & =A(\omega) x+B u, \\
x & =\left[\begin{array}{llll}
\psi_{s q} & \psi_{s d} & i_{r q} & i_{r d}
\end{array}\right]^{T}, \\
u & =\left[\begin{array}{llll}
v_{s q} & v_{s d} & v_{r q} & v_{r d}
\end{array}\right]^{T},
\end{aligned}
$$

where the state $x$ is composed of the stator flux components $\psi_{s q}, \psi_{s d}$ and the rotor current components, $i_{r q}, i_{r d}$ and the input $u$ consists of the stator voltage components, $v_{s q}, v_{s d}$ and rotor voltage components, $v_{r q}, v_{r d}$. The matrices $A(\omega)$, where $\omega$ is the rotor speed, and $B$ in (1) have the form

$$
\begin{aligned}
A(\omega) & =\left[\begin{array}{cc}
\frac{-1}{\tau_{s}} \mathcal{I}+\omega_{d q} \mathcal{J} & \frac{M}{\tau_{s}} \mathcal{I} \\
\frac{\beta}{\tau_{s}} \mathcal{I}-\omega \mathcal{J} & -\gamma \mathcal{I}+\left(\omega_{d q}-\omega\right) \mathcal{J}
\end{array}\right], \\
B & =\left[\begin{array}{cc}
\mathcal{I} & 0 \\
-\beta \mathcal{I} & \frac{1}{\sigma L_{r}} \mathcal{I}
\end{array}\right],
\end{aligned}
$$

where

$$
\mathcal{I}=\left[\begin{array}{ll}
1 & 0 \\
0 & 1
\end{array}\right], \quad \mathcal{J}=\left[\begin{array}{cc}
0 & -1 \\
1 & 0
\end{array}\right]
$$

$\gamma=\left(\frac{1-\sigma}{\sigma \tau_{s}}+\frac{1}{\sigma \tau_{r}}\right), \tau_{s}=L_{s} / R_{s}, \tau_{r}=L_{r} / R_{r}, \sigma=1-$ $M^{2} /\left(L_{s} L_{r}\right)$, and where $R_{s}, R_{r}, L_{s}, L_{r}$, are the stator and

\footnotetext{
${ }^{1}$ The dependency of the variables on time is omitted for simplicity.
}

rotor resistances and self-inductances, respectively, and $M$ is the mutual inductance.

The rotor current components $i_{r q}, i_{r d}$ and the stator voltages $v_{s q}, v_{s d}$ in (1) are the two-phase projection on the $(d, q)$ plane of, respectively, the rotor three-phase currents $i_{r R}, i_{r S}$ and $i_{r T}$ and the three-phase stator voltages $v_{s R}, v_{s S}$ and $v_{s T}$. These projections are given by the transformations [15]

$$
\left[\begin{array}{c}
i_{r q} \\
i_{r d}
\end{array}\right]=K_{r}^{d q}(\theta)\left[\begin{array}{l}
i_{r R} \\
i_{r S} \\
i_{r T}
\end{array}\right]
$$

where $K_{r}^{d q}(\theta)$ is given by

$$
\frac{2}{3}\left[\begin{array}{lll}
\cos \left(\theta_{d q}-\theta\right) & \cos \left(\theta_{d q}-\theta-\frac{2 \pi}{3}\right) & \cos \left(\theta_{d q}-\theta+\frac{2 \pi}{3}\right) \\
\sin \left(\theta_{d q}-\theta\right) & \sin \left(\theta_{d q}-\theta-\frac{2 \pi}{3}\right) & \sin \left(\theta_{d q}-\theta+\frac{2 \pi}{3}\right)
\end{array}\right],
$$

and

$$
\left[\begin{array}{l}
v_{s q} \\
v_{s d}
\end{array}\right]=K_{s}^{d q}\left[\begin{array}{l}
v_{s R} \\
v_{s S} \\
v_{s T}
\end{array}\right]
$$

where $K_{s}^{d q}$ is given by

$$
\frac{2}{3}\left[\begin{array}{lll}
\cos \left(\theta_{d q}\right) & \cos \left(\theta_{d q}-\frac{2 \pi}{3}\right) & \cos \left(\theta_{d q}+\frac{2 \pi}{3}\right) \\
\sin \left(\theta_{d q}\right) & \sin \left(\theta_{d q}-\frac{2 \pi}{3}\right) & \sin \left(\theta_{d q}+\frac{2 \pi}{3}\right)
\end{array}\right] .
$$

$\theta_{d q}=\int \omega_{d q} d t$ is the position of the reference frame and $\theta=\int \omega d t$ is the rotor position.

To implement the control law, we will assume that the rotor speed $\omega$, the rotor phase currents $i_{r R}, i_{r S}$ and $i_{r T}$ and the stator voltages $v_{s R}, v_{s S}$ and $v_{s T}$ are measured. Equations (5) and (7) then directly give the state variables $i_{r q}$ and $i_{r d}$ and input variables $v_{s d}$ and $v_{s q}$. The remaining state variables $\psi_{s q}$ and $\psi_{s d}$ in (1) will be estimated by means of flux observers.

\section{Control Strategy}

We use the control strategy presented in [5] for a standalone generation system that supplies regulated stator voltage magnitude and frequency using a DFIG. The strategy consists of two control loops designed to regulate the stator voltage magnitude and frequency. The frequency control loop consists in aligning the stator flux vector with a reference frame rotating with synchronous speed. The control of the voltage magnitude is directly done using a PI control loop.

\section{A. Stator frequency control loop}

The objective of this loop is to generate a stator voltage with constant frequency, denoted by $\omega_{r e f}$. This is achieved by using the model described in Section II with $\omega_{d q}=\omega_{\text {ref }}$ and forcing the stator flux vector to be aligned with its $d$-axis. That is, the frequency control loop aims to achieve

$$
\psi_{s q}=0, \quad \dot{\psi}_{s q}=0
$$

Note that the above guarantees that the stator flux vector effectively rotates with the desired constant frequency, $\omega_{\text {ref }}$.

The frequency control loop is implemented using a PI controller that processes the error of the $q$-component of the stator flux with respect to its desired zero reference. The 
output of the PI controller is then used to fix, or "impress", the rotor current $q$-component, $i_{r q}$, which becomes the actuation variable. That is the output of the frequency PI controller becomes the reference, $i_{r q}^{*}$, in a current control loop for the inverter

$$
i_{r q}^{*}=K_{P f} e_{s f}+K_{I f} \int e_{s f} d t
$$

where $K_{P f}, K_{I f}$ are the PI controller parameters, and

$$
e_{s f} \triangleq 0-\psi_{s q}=-\psi_{s q} \text {. }
$$

The error (11) will be, in practice, calculated using the $q$ component stator flux estimate $\hat{\psi}_{s q}$ obtained from the stator flux observer described in Section IV below.

\section{B. Stator Voltage Magnitude Control Loop}

This control loop mainly aims to reject voltage variations produced by electric load or speed changes. It is designed to regulate the magnitude, $V$, of the DFIM stator voltage vector, defined as

$$
V=\sqrt{v_{s q}^{2}+v_{s d}^{2}}
$$

where $v_{s q}$ and $v_{s d}$ are the quadrature and direct axis components referred to a synchronous reference frame, so that it tracks a constant reference $V_{\text {ref }}$. The stator voltage control loop is implemented using a PI controller

$$
i_{r d}^{*}=K_{P v} e_{s v}+K_{I v} \int e_{s v} d t,
$$

where $e_{s v}$ is the voltage error defined as

$$
e_{s v}=V_{\text {ref }}-V \text {. }
$$

The output of the voltage PI controller is used as the second reference, $i_{r d}^{*}$, in the current control loop for the inverter.

\section{Current Sensors and Flux Observers}

We consider measurement equations for the sensors that measure rotor phase currents of the form

$$
\begin{aligned}
& i_{r R, m}=i_{r R}+\eta_{R, h}, \\
& i_{r S, m}=i_{r S}+\eta_{S, h}, \\
& i_{r T, m}=i_{r T}+\eta_{T, h},
\end{aligned}
$$

where $\eta_{R, h}, \eta_{S, h}$ and $\eta_{T, h}$ are bounded ("healthy") measurement noises with zero mean value.

The rotor velocity is obtained by measuring the rotor angular position, $\theta$, so we can consider $\theta_{m}=\theta+\eta_{\theta}$ and $\omega_{m}=\omega+\eta_{\omega}$, where $\eta_{\omega}$ and $\eta_{\theta}$ are bounded measurement noises with zero mean value.

We estimate stator flux by means of three Luenberger observers, each one based on measurements from two phases. Observer 1 uses measurements (15) and (16) from phases $R$ and $S$ and computes, based on (5)-(6) and the algebraic relation $i_{r R}+i_{r S}+i_{r T}=0$,

$$
\begin{gathered}
i_{r T, 1}=-i_{r R, m}-i_{r S, m}, \\
{\left[\begin{array}{c}
i_{r q, 1} \\
i_{r d, 1}
\end{array}\right]=K_{r}^{d q}\left(\theta_{m}\right)\left[\begin{array}{c}
i_{r R, m} \\
i_{r S, m} \\
i_{r T, 1}
\end{array}\right],}
\end{gathered}
$$

where $K_{r}^{d q}\left(\theta_{m}\right)$ is of the form (6). Then, the values of $i_{r q, 1}$ and $i_{r d, 1}$ obtained in (18) are used in the observer dynamic equation

$$
\frac{d \hat{x}_{1}}{d t}=A\left(\omega_{m}\right) \hat{x}_{1}+B u+G_{K}\left(\omega_{m}\right)\left[\begin{array}{l}
\hat{i}_{r q, 1}-i_{r q, 1} \\
\hat{i}_{r d, 1}-i_{r d, 1}
\end{array}\right]
$$

where $\hat{x}_{1}=\left[\hat{\psi}_{s q, 1} \hat{\psi}_{s d, 1} \hat{i}_{r q, 1} \hat{i}_{r d, 1}\right]^{T}$ is the state estimate provided by observer 1 , and $A\left(\omega_{m}\right), B$ and $u$ are as in (1). The observer gain matrix $G_{K}\left(\omega_{m}\right)$ in (19) is such that for a given value of the measured rotor velocity $\omega_{m}$, which is fixed by an adjustable speed system, the eigenvalues of the observer closed-loop matrix $A\left(\omega_{m}\right)+G_{K}\left(\omega_{m}\right) C$, with

$$
C=\left[\begin{array}{llll}
0 & 0 & 1 & 0 \\
0 & 0 & 0 & 1
\end{array}\right],
$$

have negative real part and are proportional to those of $A(\omega)$ by a factor of $K$. That is,

$$
\operatorname{eig}\left(A(\omega)+G_{K}(\omega) C\right)=K \operatorname{eig} A(\omega) .
$$

Observer 1 passes on the following output variable to the controller whenever the switching mechanism selects this observer to implement the control law:

$$
z_{1}=\left[\hat{\psi}_{s q, 1} \hat{\psi}_{s d, 1} i_{r q, 1} i_{r d, 1}\right]^{T} .
$$

In a similar way, observer 2 uses measurements (15) and (17) from phases $R$ and $T$, and observer 3 uses measurements (16) and (17) from phases $S$ and $T$ to compute their respective stator flux estimates and corresponding output variables $z_{2}$ and $z_{3}$.

In the following section we will describe a mechanism to switch between the three observers according to a performance selection criterion. The observer that achieves the best value of the criterion will pass its output $\left(z_{1}, z_{2}\right.$ or $\left.z_{3}\right)$ to be used in the implementation of the control law.

\section{Estimate Switching Mechanism and CONTROL IMPLEMENTATION}

When the WRIM reaches a permanent regime, the $q$ component of the stator flux, $\psi_{s q}$ is (ideally) equal to zero. Thus, we could assess each observer's performance by comparing the estimate $\hat{\psi}_{s q, j}$ with zero. However, as is well known, the rotor voltages of a WRIM are PWM signals generated by the inverter that actuates the machine. These PWM signals have both a fundamental component and higher harmonic components, which result in the actual magnitude of the stator flux being the sum of a mean value and a highfrequency ripple.

We thus propose to filter the signals $\left|\hat{\psi}_{s q, j}\right|$ in order to essentially obtain their mean values. That is, for each observer we consider the following residual signal:

$$
\pi_{j} \triangleq \overline{\left|\hat{\psi}_{s q, j}\right|}, \quad j=1,2,3,
$$

where the bar over the signal denotes the respective filtered value, which is obtained by passing each signal through a low-pass filter with transfer function

$$
H(s)=\frac{1}{\left(T_{H} s+1\right)^{2}} .
$$


The design parameter $T_{H}>0$ in (24) will be chosen so that all harmonic components of the signals are sufficiently attenuated and the resulting filtered signals essentially represent their mean values.

The residual signals $\pi_{j}$ are subsequently sampled with a sampling period $T_{s}$ to obtain the discrete-time signals

$$
\pi_{j}[k]=\pi_{j}\left(k T_{s}\right), \quad k=0,1, \ldots,
$$

for $j=1,2,3$. Finally, the sampled residual signals $\pi_{j}[k]$ are compared at each sampling time $k$ according to the following switching criterion:

$$
\ell_{k}=\operatorname{argmin}_{j}\left\{\pi_{j}[k]: j \in\{1,2,3\}\right\} .
$$

At each sampling time $k$, then, the observer with index $\ell_{k}$ computed from (26) is selected by the switching mechanism and its output $z_{\ell_{k}}\left(k T_{s}\right)$ [see (22)] passed on to the controller during $k T_{s} \leq t<(k+1) T_{s}$ to substitute for the real state $x$ in the implementation of the control law.

\section{Performance Under healthy OPERATION}

In this section we will analyse the performance of the overall control scheme combining the estimate switching mechanism with the stator voltage control strategy in permanent regime and when all current sensors are operational.

In a synchronous reference frame, the electromagnetic variables of the WRIM are constant in permanent regime. In particular, assuming that the control objectives (9) and $V=V_{\text {ref }}$, with $V$ defined in (12), are achieved (and further making the simplifications $v_{s d} \approx 0$ and $R_{s} \ll R_{l}$, where $R_{l}$ is the resistance of the stator load, see [5]), we have, after some calculations, that the state $x$ of system (1) (with $\left.\omega_{d q}=\omega_{\text {ref }}\right)$ in permanent regime reaches the constant value $x=x_{\text {ref }}$ given by

$$
x_{r e f} \triangleq\left[\begin{array}{llll}
0 & \frac{V_{r e f}}{\omega_{r e f}} & -\frac{L_{s} V_{r e f}}{M R_{l}} & \frac{V_{r e f}}{M \omega_{r e f}}
\end{array}\right]^{T} .
$$

We next analyse the observer variables in permanent regime. To do so, we define the observer estimation errors as $\tilde{x}_{j} \triangleq x-\hat{x}_{j}$, that is,

$$
\tilde{x}_{j}=\left[\begin{array}{llll}
\tilde{\psi}_{s q, j} & \tilde{\psi}_{s d, j} & \tilde{i}_{r q, j} & \tilde{i}_{r d, j}
\end{array}\right]^{T}, \quad j=1,2,3,
$$

where $x$ is the state of the system (1) and $\hat{x}_{j}$ is the state estimate provided by observer $j$, for $j=1,2,3$. It is easy to show using (1), (20), observer 1 equations (18), (19) (and similar equations for observers 2 and 3 ) that, under healthy operation of all current sensors, the estimation errors (28) satisfy the dynamic equations

$$
\dot{\tilde{x}}_{j}=A_{G} \tilde{x}_{j}+B_{G}(x)\left[\begin{array}{c}
\eta_{\omega} \\
\tilde{\eta}_{j}
\end{array}\right], \quad j=1,2,3,
$$

where we have used $\omega_{m}=\omega+\eta_{\omega}$ and the definitions

$$
\begin{aligned}
A_{G} & \triangleq A\left(\omega_{m}\right)+G_{K}\left(\omega_{m}\right) C \\
B_{G}(x) & \left.\triangleq\left[\begin{array}{lr}
0 \mathcal{I} & 0 \mathcal{I} \\
\mathcal{J} & \mathcal{J}
\end{array}\right] x \quad G_{K}\left(\omega_{m}\right)\right] \\
\tilde{\eta}_{j} & \triangleq K_{r}^{d q}\left(\theta_{m}\right) \eta_{j},
\end{aligned}
$$

with $\mathcal{I}, \mathcal{J}$ as in (4), $\eta_{1}=\left[\begin{array}{lll}\eta_{r R, h} & \eta_{r S, h} & -\eta_{r R, h}-\eta_{r S, h}\end{array}\right]^{T}$, $\eta_{2}=\left[\begin{array}{lll}\eta_{r R, h} & -\eta_{r R, h}-\eta_{r T, h} & \eta_{r T, h}\end{array}\right]^{T}$ and $\eta_{3}=\left[\begin{array}{l}-\eta_{r S, h}- \\ \eta_{r, h}\end{array}\right.$ $\left.\eta_{r T, h} \quad \eta_{r S, h} \quad \eta_{r T, h}\right]$.

Assume that a permanent regime has been reached where the state $x$ takes the constant value $x=x_{r e f}$ given by (27). Since, for a fixed $\omega_{m}$, the matrix $A_{G}$ defined in (30) is stable by design [see discussion after (19), in particular equation (21)], the matrix $K_{r}^{d q}\left(\theta_{m}\right)$ has bounded entries [see (6)], and the noises $\eta_{\omega}, \eta_{j}, j=1,2,3$ are bounded by assumption, the states of (29) will be ultimately bounded. In particular, the $q$ components of the stator flux estimation errors are ultimately bounded, namely,

$$
\left|\tilde{\psi}_{s q, j}\right| \leq \epsilon_{j}
$$

for $j=1,2,3$, for some $\epsilon_{j}>0$. This bound can be used, in turn, to obtain ultimate bounds for the observer error signals (23):

$$
\pi_{j}=\overline{\left|\hat{\psi}_{s q, j}\right|}=\overline{\left|\psi_{s q, j}-\tilde{\psi}_{s q, j}\right|}=\overline{\left|\tilde{\psi}_{s q, j}\right|} \leq\left|\tilde{\psi}_{s q, j}\right| \leq \epsilon_{j}
$$

for $j=1,2,3$. Thus, the sampled error signal $\pi_{j}[k], j=$ $1,2,3$, defined in (25), satisfies the same bounds, that is,

$$
\pi_{j}[k] \leq \epsilon_{j}
$$

If the bounds on the noises are small, then the bounds (34) on the sampled observer error signals under healthy operation will also be small. As we will show in Section VII below, this is in stark contrast with the bounds that these observer error signals have when a current sensor associated with the corresponding observer fails. This difference in bounds between healthy and faulty operation is the key to achieve fault tolerance in the proposed approach.

\section{PERFORMANCE UNDER CURRENT SENSOR FAULT AND FAULT TOLERANCE PRINCIPLE}

In this section we analyse the performance of the switching control scheme under abrupt faults of current sensors. We will model an abrupt fault as an instantaneous change in one of the sensor measurement equations, from (15)-(17) to

$$
i_{r j, m}=\eta_{j, f}, \quad j \in\{R, S, T\},
$$

where $\eta_{R, f}, \eta_{S, f}, \eta_{T, f}$ are bounded ("under-fault") measurement noises. We will assume that only one sensor can fail at the time.

Consider then a fault modelled by (35) in, for example, the sensor that measures the phase current $i_{r R}$. Note from Section IV that only observers 1 and 2 will be affected by this fault whereas observer 3 will remain unaffected. Similarly, a fault in the sensor that measures the phase current $i_{r S}$ affects only observers 1 and 3, and a fault in the sensor that measures the phase current $i_{r T}$ affects only observers 2 and 3 .

Substituting (35) in the equations of the observers affected by a fault of type $F \in\{R, S, T\}$ (that is, a fault in the sensor that measures the phase current $i_{r F}$ ) we have, after some calculations, that the estimation errors in permanent regime change their dynamics from (29) to

$$
\dot{\tilde{x}}_{l}=A_{G} \tilde{x}_{l}+B_{G}(x)\left[\begin{array}{l}
\eta_{\omega} \\
\tilde{\eta}_{l}^{F}
\end{array}\right]+G_{K}\left(\omega_{m}\right) K_{r}^{d q}\left(\theta_{m}\right) E_{l}^{F} i_{r F},
$$


for $l \in \mathcal{L}^{F}$, where $\mathcal{L}^{F}$ denotes the set of indices of the observers affected by the fault $F$. The set $\mathcal{L}^{F}$, the vectors $E_{l}^{F}$, for $l \in \mathcal{L}^{F}$, and the input $i_{r F}$ change according to the type of fault as described in the table:

\begin{tabular}{|c|c|c|c|}
\hline Fault & $\mathcal{L}^{F}$ & $E_{l}^{F}, l \in \mathcal{L}^{F}$ & $i_{r F}$ \\
\hline$R$ & $\{1,2\}$ & $E_{1}^{R}=-\left[\begin{array}{c}-1 \\
0 \\
1\end{array}\right], E_{2}^{R}=\left[\begin{array}{c}-1 \\
1 \\
0\end{array}\right]$ & $i_{r R}$ \\
\hline$S$ & $\{1,3\}$ & $E_{1}^{S}=\left[\begin{array}{c}0 \\
-1 \\
1\end{array}\right], E_{3}^{S}=\left[\begin{array}{c}1 \\
-1 \\
0\end{array}\right]$ & $i_{r S}$ \\
\hline$T$ & $\{2,3\}$ & $E_{2}^{T}=\left[\begin{array}{c}0 \\
1 \\
-1\end{array}\right], E_{3}^{T}=\left[\begin{array}{c}1 \\
0 \\
-1\end{array}\right]$ & $i_{r T}$ \\
\hline
\end{tabular}

The "under-fault" noise input $\eta_{l}^{F}$ in (36) is a combination of measurement noises; for example, for a fault in the sensor that measures the phase current $i_{R}$, these inputs take the form $\eta_{1}^{R}=\left[\begin{array}{lll}\eta_{R, f} & \left.\eta_{S, h}-\eta_{R, f}-\eta_{S, h}\right)\end{array}\right]^{T}, \eta_{2}^{R}=$ $\left[\begin{array}{lll}\eta_{R, f} & \left.-\eta_{R, f}-\eta_{T, h}\right) & \eta_{T, h}\end{array}\right]^{T}$. Assuming that the state $x$ preserves the permanent regime value $x=x_{\text {ref }}$ (this will be guaranteed if the fault tolerance conditions hold, see below) then (36) is a stable linear system driven by two bounded external inputs: the "under-fault" noise input $\left[\eta_{\omega}\left(\tilde{\eta}_{l}^{F}\right)^{T}\right]^{T}$, and the phase current $i_{r F}$ projected by the matrix $K_{r}^{d q}\left(\theta_{m}\right)$,

We thus observe from (36) that, if a fault of type $F$ occurs, the estimation errors of the affected observers (that is, those with indices in $\mathcal{L}^{F}$ ) will have a component which is proportional to the phase current measured by the failed sensor (typically of much larger amplitude than the measurement noise bounds), and thus the error signals (23), as well as their sampled values (25), will show an offset away from zero and an associated lower bound of the form

$$
\pi_{l}^{F}[k] \geq \epsilon_{l}^{F}, \quad l \in \mathcal{L}^{F} .
$$

Under healthy operation, the sampled error signals $\pi_{j}[k]$, defined in (25), on which the switching strategy bases its decision (26) at each sampling time, are upper bounded as in (34). On the other hand, under a current sensor fault, the sampled error signals $\pi_{l}^{F}[k]$ associated with the two observers affected by the fault are lower bounded as in (37). Thus, the scheme with switching criterion (26) will be fault tolerant under a fault of type $F$, where $F \in\{R, S, T\}$ indicates a fault in the sensor measuring the phase current $i_{R}$, $i_{S}$ or $i_{T}$, if

$$
\epsilon_{l}^{F}>\epsilon_{j}, \quad \text { for } \quad l \in \mathcal{L}^{F} \text { and } \quad j \in\{1,2,3\} \backslash \mathcal{L}^{F} .
$$

Note that, if conditions (38) hold, then observer $j, j \in$ $\{1,2,3\} \backslash \mathcal{L}^{F}$, will be chosen by the switching criterion (26) over observers $l, l \in \mathcal{L}^{F}$, which are the ones affected by the faulty sensor

\section{Simulation Results}

In this section we present simulation results to illustrate the fault tolerance properties of the proposed scheme. The
DFIG has the parameters and reference values listed in the table:

\begin{tabular}{|c|c|c|c|}
\hline Rated & values & $\begin{array}{c}\text { Parameters } \\
\text { and reference }\end{array}$ & values \\
\hline$P$ & $5.5[\mathrm{KW}]$ & $V_{\text {ref }}$ & $311 \mathrm{~V}$ \\
\hline$v_{s}$ & $220[\mathrm{~V}]$ & $L_{s}=L_{r}$ & $122.8 \mathrm{mH}$ \\
\hline$v_{r}$ & $132[\mathrm{~V}]$ & $M$ & $121 \mathrm{mH}$ \\
\hline$i_{s}$ & $12[\mathrm{~A}]$ & $R_{s}$ & $0.67 \Omega$ \\
\hline$i_{r}$ & $16[\mathrm{~A}]$ & $R_{r}$ & $1.17 \Omega$ \\
\hline$\psi_{s}$ & $1[\mathrm{Vs}]$ & $\omega_{r e f}$ & $314 \mathrm{rad} / \mathrm{s}$ \\
\hline
\end{tabular}

The rotor speed is assumed fixed by an adjustable speed system at $\omega=0.8 \omega_{\text {ref }}$. We assume a speed sensor noise of $0.5 \%$. The current sensor noises in (15)-(17) are bounded as $\left|\eta_{R, h}\right| \leq 0.1 \mathrm{~A},\left|\eta_{S, h}\right| \leq 0.1 \mathrm{~A}$ and $\left|\eta_{T, h}\right| \leq 0.1 \mathrm{~A}$. The same bound of $0.1 \mathrm{~A}$ is used for the "under-fault" noises in $(35)$.

The parameters of the PI controller of the frequency control loop [see (10)] are $K_{P f}=70$ and $K_{I f}=10^{5}$, and those of the voltage control loop [see (10)] are $K_{P v}=0.01$ and $K_{I v}=100$. The observers gain $G_{K}(\omega)$, satisfies (21) with $K=2$. The sampling time is taken as $T_{s}=0.1 \mathrm{~ms}$ and the parameter $T_{H}$ in (24) is $T_{H}=0.02$.

To simulate a realistic implementation of the controller, the control signals (phase currents) given by the controller are passed through a three phase inverter which gives the phase voltages that feed the induction motor. We have selected an inverter that implements a current control method with a sampling frequency of $20 \mathrm{KHz}$. The simulation scenario is as follows. The reference signal for stator voltage is a step that starts at $t=0 \mathrm{~s}$. The load of the DFIM is pulsating as shown in Figure 3 (bottom-right plot). At $t=1.07 \mathrm{~s}$ a fault in the sensor that measures the phase current $i_{T}$ occurs, that is, its measurement equation changes from (15) to (35).

The top plot of Figure 2 shows the switching signal $\ell_{k}$ resulting from the switching mechanism decision (26). Notice that after the fault at $t=1.07 \mathrm{~s}$ the switching mechanism only selects observer 1 , thus achieving fault tolerance. Plots 2 to 4 of Figure 2 show the sampled error signals $\pi_{j}[k]$, $j=1,2,3$, defined in (25), corresponding to observers 1,2 and 3, respectively. As analysed in Section VII, after the fault at $t=1.07 \mathrm{~s}$ the error signals for observers 2 and 3 move quickly to values noticeably away from zero, whereas the error signal for observer 1 maintains the same small values (near zero) as before the occurrence of the fault.

Figure 3 shows the $d q$-components of the stator voltage $V \approx V_{\text {ref }}=311 \mathrm{~V}$ (top-left), the stator flux components, $\psi_{s d}$ and $\psi_{s q}$ (top-right), the rotor currents $i_{r d}, i_{r q}$ (bottomleft), and the load torque $\tau_{l}$ (bottom-right), under the fault tolerant control scheme. Note that the simulated fault at $t=1.07 \mathrm{~s}$ has no noticeable impact on these responses and thus, the controller achieves the desired objective, even with a pulsating load which, in turn, illustrates the robustness of the method under load variations.

We conclude this section with a brief discussion on further robustness properties of the proposed fault tolerant control scheme. In addition to implicitly testing the robustness of 

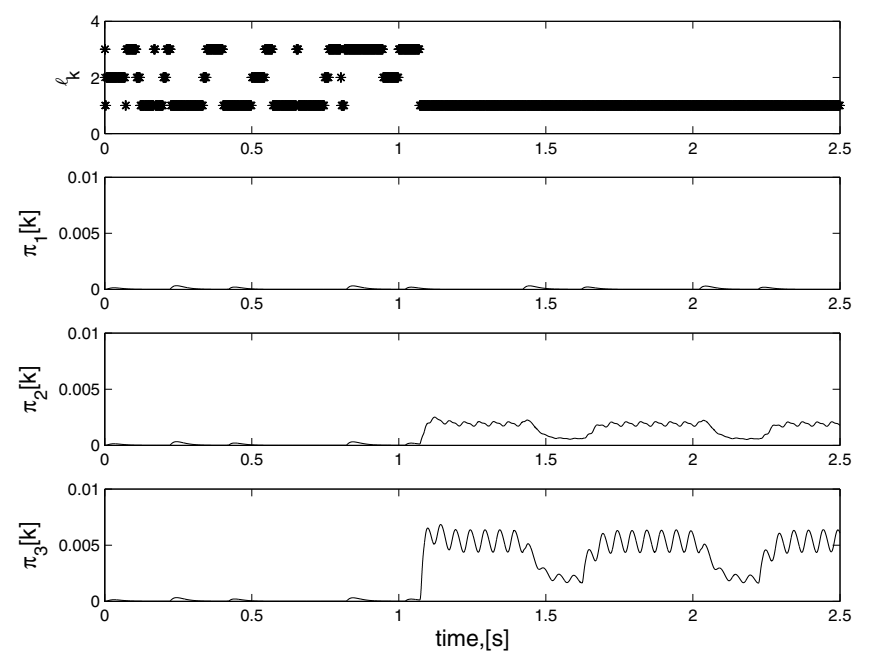

Fig. 2. Switching sequence and observer sampled error signals. Note that after the fault at $t=1.07 \mathrm{~s}$ the switching scheme only selects observer 1 .
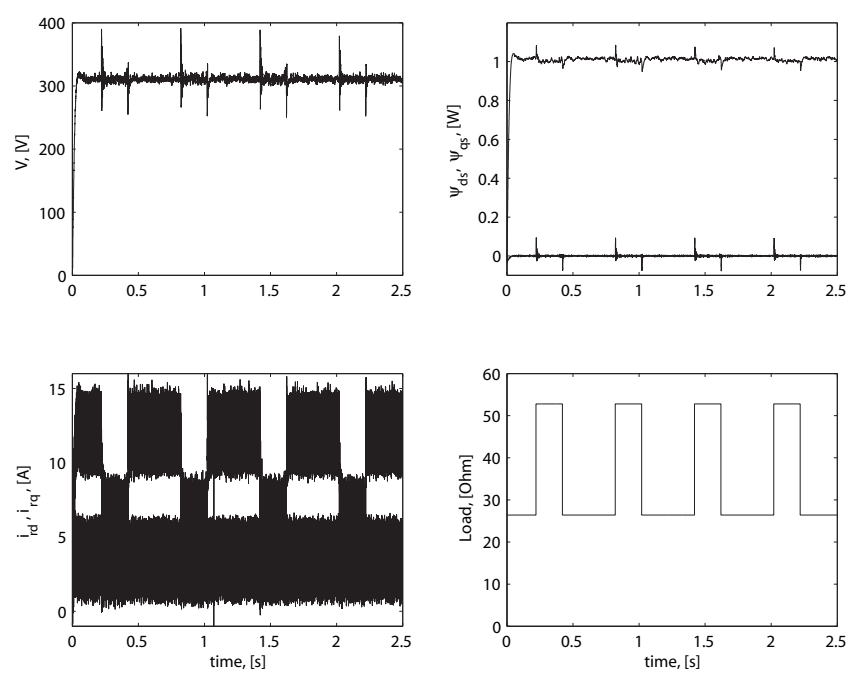

Fig. 3. Stator voltage $V \approx V_{\text {ref }}=311 \mathrm{~V}$ (top-left), stator flux components, $\psi_{s d}, \psi_{s q}$ (top-right), rotor currents $i_{r d}, i_{r q}$ (bottom-left), and load resistance $R_{l}$ (bottom-right).

the scheme to the inverter induced ripple, as shown in the presented simulation study, we have performed further tests including parameter variations in the WRIM model. More specifically, the simulation scenario presented above has been repeated after increasing by $20 \%$, one by one and all simultaneously, the following parameters: $R_{s}, R_{r}$ and the leakage inductances $L_{s}-M, L_{r}-M$. The results of those studies of parameter variations showed that the scheme continued to perform satisfactorily and preserved the fault tolerance capabilities in all tested cases.

\section{CONCLuSIONS}

We have proposed a multisensor switching strategy for fault tolerant control of rotor wound induction motors operating as stand alone generators. The proposed strategy combines three current sensors and associated observers that estimate the stator flux. The estimates provided by the observers are compared at each sampling time by a switching mechanism, which selects the sensors-observer pair with the smallest error between the estimated flux magnitude and a desired flux reference. The estimates provided by the selected pair are used to implement a vector control law. The switching selection is such that measurements from faulty current sensors are automatically avoided by the switching mechanism, thus maintaining good performance levels even in the presence of a faulty sensor. Simulation results under realistic conditions illustrate the effectiveness of the scheme. Robustness studies of parameter variations have shown that the scheme continued to perform satisfactorily and preserved the fault tolerance capabilities in all tested cases.

\section{REFERENCES}

[1] J. Martínez, X. Zhuo, J. De Doná, and M. Seron, "Multisensor switching strategy for automotive longitudinal control," in Proc. of the 2006 American Control Conference, Minneapolis, MN, USA, 2006.

[2] M. Seron, X. Zhuo, J. De Doná, and J. Martínez, "Multisensor switching control strategy with fault tolerance guarantees," Automatica, vol. 44 , no. 1 , pp. $88-97,2008$.

[3] S. Müller, M. Deicke, and R. W. Doncker, "Doubly fed induction generators systems for wind turbines," IEEE Industry Application Magazine, vol. 8, no. 3, pp. 26-33, 2002.

[4] K. Rothenhagen and F. W. Fuchs, "Current sensor fault detection by bilinear observer for a doubly fed induction generator," in The 32nd Annual Conference of the IEEE Industrial Electronics Society, IECON'06, Paris, France, 2006.

[5] D. Forchetti, G. García, and M. I. Valla, "Adaptive observer for sensorless control of stand alone doubly fed induction generator," IEEE Trans. on Industrial Electronic, vol. 56, no. 10, pp. 4174-4180, 2009.

[6] G. Verghese and S. Sanders, "Observers for flux estimation in induction machines," IEEE Transactions on Industrial Electronics, vol. 35, no. 1, February 1988.

[7] M. Galvez-Carrillo and M. Kinnaert, "Fault detection and isolation in current and voltage sensors of doubly-fed induction generators," in Proceedings of the 7th IFAC Symposium on Fault Detection, Supervision and Safety of Technical Processes, Barcelona, Spain, June 2009.

[8] S. Karimi, A. Gaillard, P. Poure, and S. Saadate, "Current sensor faulttolerant control for WECS with DFIG," IEEE Trans. on Industrial Electronic, vol. 56, no. 10, pp. 4660-4669, 2009.

[9] K. Rothenhagen and F. W. Fuchs, "Performance of diagnosis methods for igbt open circuit faults in three phase voltage source inverters for ac variable speed drives," in EPE05, Dresden, 2005.

[10] — "Advanced sensor fault detection and control reconfiguration of wind power plants using doubly fed induction generators," in Power Electronics Specialists Conference, 2008. PESC 2008. IEEE, Island of Rhodes, Greece, 2008.

[11] — - "Current sensor fault detection, identification, and reconfiguration for doubly fed induction generators," in The 33nd Annual Conference of the IEEE Industrial Electronics Society, IECON'07, Taipei, Taiwan, 2007.

[12] M. Seron, M. Romero, and J. De Doná, "Sensor fault tolerant control of induction motors," in Proceedings of the 17th IFAC World Congress, Seoul, July 2008.

[13] M. Romero, M. Seron, and J. De Doná, "Sensor fault tolerant direct torque and flux control of induction motors," in 7th IFAC Symposium on Fault Detection, Supervision and Safety of Technical Processes, SAFEPROCESSO9, Barcelona, Spain, 2009.

[14] M. Romero and M. Seron, "Speed-sensorless control of induction motors with improved fault tolerance against current sensor failure," in in 18th Mediterranean Conference on Control and Automation, Marrakech, Morocco, June 23-25 2010.

[15] P. Krause, O. Wasynczuk, and S. Sudhoff, Analysis of Electric Machinery. New York: IEEE press, 1995. 